\title{
Nonmotor symptoms predict quality of life in patients with early Parkinson disease
}

Nonmotor symptoms of Parkinson disease (PD), such as fatigue, depression and pain, are the most important factors contributing to poor health-related quality of life in patients with early Parkinson disease, according to Bernd Müller, investigator in the Norwegian ParkWest population-based cohort study.

Nonmotor and motor symptoms are both thought to have key roles in the perceived quality of life in mid-stage $\mathrm{PD}$, but the impact of nonmotor symptoms in patients with early PD has received little research attention, and current therapeutic approaches focus on alleviating patients' motor symptoms.

Müller and colleagues assessed the clinical symptoms and perceived healthrelated quality of life of 166 individuals at the time of diagnosis with PD and again 3 years later. Problems with gait control and taking care of personal needs were the most important motor symptoms affecting health-related quality of life. "A reduced ability to walk and conduct personal needs threatens an individual's independence," notes Müller. These problems should be taken seriously in patients with early PD, he stresses, "even if they seem be able to cope with their symptoms."

However, nonmotor symptomsparticularly the severity of fatigue, depression and pain-had a stronger correlation with both mental and physical domains of quality of life than did motor symptoms. "While motor symptoms

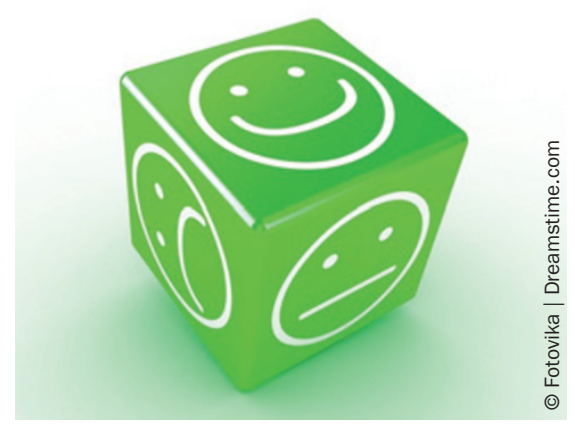

predominantly impair physical quality of life, nonmotor symptoms affect both physical and mental quality-of-life measurements," summarizes Müller. As the disease progressed, fatigue became an increasingly important contributor to impaired quality of life. "Fatigue seems to be an often neglected symptom in both the clinic and research, despite its clear impact on quality of life," he reflects.

The team intend to repeat their analyses in patients 7-9 years after diagnosis of PD. "We are following the cohort of PD patients and their relatives in the ParkWest study and we may also look at the impact of the various PD-related symptoms on relatives," explains Müller.

\section{Hemi Malkki}

Original article Müller, B. et al. Importance of motor vs. non-motor symptoms for health-related quality of life in early Parkinson's disease. Parkinsonism Relat. Disord. doi:10.1016/j.parkreldis.2013.07.010 